Pacific Journal of Mathematic 


\section{RELATIONS AMONG GENERALIZED MATRIX FUNCTIONS}

\section{RUSSELl MERRIS}

Let $G$ be a permutation group of degree $m$. Let $\lambda$ be an irreducible, complex character of $G$. If $A=\left(a_{i j}\right)$ is an $m$ by $m$ matrix, the generalized matrix function of $A$ corresponding to $G$ and $\lambda$ is defined by

$$
d_{\lambda}^{G}(A)=\sum_{\sigma \in G} \lambda(\sigma) \prod_{t=1}^{m} a_{t \sigma(t)} .
$$

We obtain relations among generalized matrix functions arising from $G$ and those arising from a subgroup $H$ of $G$. The methods yield some information about the corresponding symmetry classes of tensors.

Generalized matrix functions were invented by I. Schur to improve E. Fischer's improvement of the Hadamard determinant theorem. Specifically, Schur proved that

$$
d_{\lambda}^{G}(A) \geqq \lambda(i d) \operatorname{det}(A)
$$

for all positive semidefinite Hermitian matrices $A$ (write $A \geqq 0$ ).

1. The main results. In what has become a classic paper on the subject, S. G. Williamson obtained the following result in [14]: If $H$ is a subgroup of $G$ and if $\lambda$ is a character of $G$ of degree 1, then

$$
d_{\lambda}^{G}(A) \leqq[G: H] d_{\lambda}^{H}(A)
$$

for all $A \geqq 0$. In [6], the present author improved Williamson's result as follows: Let $H$ be a subgroup of $G$. Let $\lambda$ be an irreducible character of $G$. Suppose the restriction of $\lambda$ to $H$ is $\lambda(i d) \chi / \chi(i d)$ for some irreducible character $\chi$ of $H$ (i.e., suppose $\left.\lambda\right|_{H}$ is a multiple of $\chi$ ). Then

$$
\lambda(i d) d_{\lambda}^{G}(A) \leqq[G: H] \chi(i d) d_{\chi}^{H}(A)
$$

for every $A \geqq 0$. 
The inequality (2) has further been improved [7, Corollary 2]: Let $H$ be a subgroup of $G$. Let $\chi$ be an irreducible character of $H$. If $A \geqq 0$, then

$$
\Sigma \eta(i d) d_{\eta}^{G}(A) \leqq[G: H] \chi(i d) d_{\chi}^{H}(A)
$$

where the summation is over those irreducible characters $\eta$ of $G$ whose restriction to $H$ is a multiple of $\chi$. Our first result is an upper bound for $[G: H]_{\chi}(i d) d_{\chi}^{H}(A)$ to go along with (3).

THEOREM 1. Let $H$ be a subgroup of $G$. Let $\chi$ be an irreducible character of $H$. If $A \geqq 0$, then

$$
[G: H] \chi(i d) d_{\chi}^{H}(A) \leqq \Sigma \eta(i d) d_{\eta}^{G}(A)
$$

where the summation is over those irreducible characters $\eta$ of $G$ whose restriction to $H$ contains $\chi$ as a component. Moreover, equality holds for all $A \geqq 0$, if and only if $\left.\eta\right|_{H}$ is a multiple of $\chi$ whenever $\chi$ is a component of the restriction of $\eta$ to $H$ (i.e., if and only if $(\eta, \chi)_{H} \neq 0$ implies $\left.\eta\right|_{H}=$ $\eta(i d) \chi / \chi(i d))$.

Observe that the case of equality in (4) is sufficient for equality to hold in (3). At the end of this section, we will show that it is also necessary, i.e., the case of equality for (3) is the same as that for (4). In [9, Theorem 8] a class of examples is given in which equality holds in (3)-(4).

It is worth mentioning that the situation is considerably simpler if $H$ is assumed to be normal in $G$. In that case, if $\left.\lambda\right|_{H}$ is a multiple of $\chi$, then $\chi$ is invariant under conjugation by elements of $G$. By Clifford's theorems [2, p. 53] if $\chi$ is invariant under conjugation by $G$ and if $\left.\chi \in \eta\right|_{H}$, then $\left.\eta\right|_{H}=\eta($ id $) \chi / \chi(i d)$. Therefore, if $H \Delta G$ then either the summation on the left of (3) is vacuous, or equality holds in (3)-(4). Explicitly, if $H \Delta G$ and if $\chi$ is an irreducible character of $H$ invariant under conjugation by $G$, then

$$
[G: H] \chi(i d) d_{\chi}^{H}(A)=\Sigma \eta(i d) d_{\eta}^{G}(A)
$$

where the summation is over those irreducible characters $\eta$ of $G$ whose restriction to $H$ contains $\chi$ as a component. This seems a better result than [7, Corollary 1].

Our second result is an improvement of (2) in a direction different from (3). 
THEOREM 2. Let $H$ be a subgroup of $G$. Let $\lambda$ be an irreducible character of $G$. Suppose $\left.\lambda\right|_{H}=a_{1} \chi_{1}+\cdots+a_{r} \chi_{r}$, where $a_{1}, \cdots, a_{r}$ are positive integers and $\chi_{1}, \cdots, \chi_{r}$ are the distinct irreducible components of the restriction of $\lambda$ to $H$. Then

$$
\lambda(i d) d_{\lambda}^{G}(A) \leqq[G: H] \sum_{i=1}^{r} \chi_{\imath}(i d) d_{\chi_{i}}^{H}(A)
$$

for all $A \geqq 0 . \quad$ Equality holds for all $A \geqq 0$, if and only if none of the $\chi_{1}$ appears in the restriction to $H$ of an irreducible character of $G$ different from $\lambda$ (i.e., if and only if $\left(\eta, \chi_{i}\right)_{H}=0,1 \leqq i \leqq r$, for evey irreducible character $\eta$ of $G$ different from $\lambda$ ).

Of course, the case $r=1$ is (2).

Before getting involved in the proofs, we illustrate the results with some examples.

EXAMPLE 1. Let $G=S_{3}$, the full symmetric group of degree 3. Let $\lambda$ be the irreducible character of $G$ of degree 2. (Then $\lambda(\sigma)$ is one less than the number of fixed points of $\sigma$.) Let $H=A_{3}$, the alternating group. Then $\left.\lambda\right|_{H}=\chi_{1}+\chi_{2}$, where $\chi_{1}(123)=\exp (2 \pi i / 3)=\omega, \chi_{2}(123)=$ $\omega^{2}$.

Let

$$
M=\left[\begin{array}{rrr}
2 & -1 & -1 \\
-1 & 2 & -1 \\
-1 & -1 & 2
\end{array}\right]
$$

Then $d_{\lambda}^{G}(M)=18$, and $d_{\chi_{1}}^{H}(M)=d_{\chi_{2}}^{H}(M)=9$. Plugging into (5), we obtain $2(18) \leqq 2(9+9)$, equality. Indeed, the other irreducible characters of $S_{3}$ are the identically 1 character, and the alternating character $\epsilon$. The restriction of either of these to $A_{3}$ is the identically 1 character which, of course, contains neither $\chi_{1}$ nor $\chi_{2}$. Of course, (2) is not applicable at all, and (3) merely reduces to the statement that $d_{\chi_{1}}^{H}(M)$ and $d_{\chi_{2}}^{H}(M)$ are nonnegative. Equation (4) becomes

$$
[G: H] \chi_{1}(i d) d_{\chi_{1}}^{H}(M) \leqq \lambda(i d) d_{\lambda}^{G}(M),
$$

which is precisely the reverse of what one might have expected given only (2).

If we were to take the same $G$ and $H$ but begin with $\chi$ identically one on $H$, then (3)-(4) become 


$$
2 d_{1}^{H}(A)=\operatorname{per}(A)+\operatorname{det}(A)
$$

for all $A \geqq 0$. In fact, of course, (7) holds for all 3 by 3 matrices $A$. (Indeed, it can be shown, using Lemma 3(a) below, that if equality holds in (3)-(4) for all $A \geqq 0$, it holds for all $m$-square matrices $A$.)

EXAMPLE 2. Let $G^{*}=S_{3}$. Let $\lambda$ be the irreducible character of $G$ of degree 2. Let $H=\{i d,(13)\}$. Then $\left.\lambda\right|_{H}=1+\epsilon$. If we use the matrix $M$ given in (6), we obtain $d_{\lambda}^{G}(M)=18, d_{1}^{H}(M)=10$, and $d_{\epsilon}^{H}(M)=6$. Equation (5) yields $2(18) \leqq 3(10+6)$. Equation (4) produces

$$
\begin{aligned}
& 3(10) \leqq 2(18)+\operatorname{per}(M) \\
& 3(6) \leqq 2(18)+\operatorname{det}(M) .
\end{aligned}
$$

EXAMPLE 3. Let $G=S_{3}$. Let $\lambda$ be the irreducible character of $G$ of degree 2. Let $H=\{i d\}$. Then $\left.\lambda\right|_{H}=1+1$, i.e., the restriction of $\lambda$ to $H$ is a multiple of the identically 1 character. In this case, (5) collapses to (2), and (3)-(4) become equality [8, Corollary 1].

We end this section by determining the case of equality in (3). As we have observed, if $\left.\eta\right|_{H}$ is a multiple of $\chi$ whenever $\chi$ is a component of $\left.\eta\right|_{H}$ then equality holds in (4), but in this case the right hand side of (4) is the left hand side of (3). Suppose, then, that equality holds in (3) for all $A \geqq 0$. Let $\chi^{*}$ be the character of $G$ induced by $\chi[2]$. Let $N$ be the set of irreducible characters $\eta$ of $G$ whose restriction to $H$ is a multiple of $\chi$. Comparing degrees, we have

$$
\sum_{\eta \in N}\left(\eta, \chi^{*}\right)_{G} \eta(i d) \leqq \chi^{*}(i d)
$$

with equality if and only if $\left(\lambda, \chi^{*}\right)_{G}=0$ for those irreducible characters $\lambda$ of $G$ which do not belong to $N$. But, by the Frobenius Reciprocity Theorem, $\left(\lambda, \chi^{*}\right)_{G}=(\lambda, \chi)_{H}$ for every irreducible character $\lambda$ of $G$. It suffices, therefore, to prove that equality holds in (10). Now, we know that $\chi^{*}(i d)=[G: H] \chi(i d)$, and, of course, $(\eta, \chi)_{H}=\eta($ id $) / \chi(i d)$ for $\eta \in N$. Plugging into (10) we have

$$
\sum_{\eta \in N}[\eta(i d) / \chi(i d)] \eta(i d) \leqq[G: H] \chi(i d),
$$

and we are attempting to show that equality holds. Letting $A=$ the identity in (3), and assuming equality, we obtain

$$
\sum_{\eta \in N} \eta(i d)^{2}=[G: H] \chi(i d)^{2}
$$


2. Symmetry classes of tensors. Let $V$ be a complex inner product space of dimension $n$. Let $\otimes^{m} V$ denote the $m$ th tensor power of $V$ and write $v_{1} \otimes \cdots \otimes v_{m}$ for the tensor product of $v_{1}, \cdots, v_{m} \in$ $V$. The inner product on $V$ induces an inner product on $\otimes^{m} V$ which is determined by the formula

$$
\left(v_{1} \otimes \cdots \otimes v_{m}, w_{1} \otimes \cdots \otimes w_{m}\right)=\prod_{t=1}^{m}\left(v_{t}, w_{t}\right) .
$$

For $\sigma \in S_{m}$, the symmetric group, let $P\left(\sigma^{-1}\right)$ be the linear operator on $\otimes^{m} V$ whose action is determined by

$$
P\left(\sigma^{-1}\right) v_{1} \otimes \cdots \otimes v_{m}=v_{\sigma(1)} \otimes \cdots \otimes v_{\sigma(m)}
$$

for all $v_{1}, \cdots, v_{m} \in V$. It follows that $\sigma \rightarrow P(\sigma)$ is a representation of $S_{m}$.

The adjoint, $P(\sigma)^{*}$, of $P(\sigma)$ with respect to the induced inner product is easily seen to be $P\left(\sigma^{-1}\right)=P(\sigma)^{-1}$.

Let $G$ be a subgroup of $S_{m}$. Let $\mathscr{I}(G)$ denote the set of irreducible (complex) characters of $G$. If $\lambda \in \mathscr{I}(G)$, define

$$
T(G, \lambda)=\frac{\lambda(i d)}{o(G)} \sum_{\sigma \in G} \lambda(\sigma) P(\sigma) .
$$

It is known ([13], [11] or [3]) that $\{T(G, \lambda): \lambda \in \mathscr{I}(G)\}$ is a set of pairwise annihilating (with respect to the induced inner product they are hermitian) idempotents which sum to $1_{\otimes}$, the identity operator on $\otimes^{m} V$.

Lemma 1. Let $H$ be a subgroup of $G$. Let $\lambda \in \mathscr{I}(G)$ and $\chi \in$ $\mathscr{I}(H)$. Then $T(H, \chi)$ and $T(G, \lambda)$ commute.

Proof.

$$
\begin{aligned}
& \frac{o(G) o(H)}{\lambda(\text { id) } \chi(i d)} T(H, \chi) T(G, \lambda) \\
& =\sum_{\pi \in H} \sum_{\sigma \in G} \chi(\pi) \lambda(\sigma) P(\pi \sigma) \\
& =\sum_{\sigma \in G} \sum_{\pi \in H} \chi(\pi) \lambda\left(\pi^{-1} \sigma\right) P(\sigma) \\
& =\sum_{\sigma \in G} \sum_{\pi \in H} \lambda\left(\sigma \pi^{-1}\right) \chi(\pi) P(\sigma) \\
& =\sum_{\sigma \in G} \sum_{\pi \in H} \lambda(\sigma) \chi(\pi) P(\sigma \pi) .
\end{aligned}
$$


LeMma 2. Let $H$ be a subgroup of $G$. Let $\lambda \in \Phi(G)$ and $\chi \in$ $\mathscr{I}(H)$. If $m \leqq n$, then $T(H, \chi) T(G, \lambda)$ is zero if and only if $\left.\chi \notin \lambda\right|_{H}$.

Proof. Let $\sigma \rightarrow L(\sigma)=\left(\ell_{i j}(\sigma)\right)$ be an irreducible representation of $G$ which affords $\lambda$. Assume the restriction of $L$ to $H$ is fully reduced. Define

$$
T_{i}=\frac{\lambda(i d)}{o(G)} \sum_{\sigma \in G} \ell_{i i}(\sigma) P(\sigma) .
$$

Of course, $T(G, \lambda)=\Sigma T_{t}$. Observe

$$
\begin{aligned}
T(H, \chi) T_{i} & =\frac{\chi(i d) \lambda(i d)}{o(H) o(G)} \sum_{\pi \in H} \sum_{\sigma \in G} \chi(\pi) \ell_{i i}\left(\pi^{-1} \sigma\right) P(\sigma) \\
& =\frac{\lambda(i d)}{o(G)} \sum_{\sigma \in G} \sum_{k=1}^{\lambda(d)} \ell_{k i}(\sigma)\left\{\frac{\chi(i d)}{o(H)} \sum_{\pi \in H} \chi(\pi) \ell_{i k}\left(\pi^{-1}\right)\right\} P(\sigma) .
\end{aligned}
$$

By the orthogonality relations $([10, \S 9]$ or $[2$, Ch. $1, \S 1])$, the term in curly brackets is zero unless the component of $\left.L\right|_{H}$ which contains position $i, i$ affords $\chi$, in which case the bracketed term is $\delta_{t k}$. In particular, $T(H, \chi) T_{i}=0$ for all $i$ and hence $T(H, \chi) T(G, \lambda)=0$, if $(\chi, \lambda)_{H}=0$. If $(\chi, \lambda)_{H} \neq 0$, then since $\left.L\right|_{H}$ is fully reduced, it contains a component which affords $\chi$. Suppose such a component lies in rows and columns $t+1, t+2, \cdots, t+\chi(i d)$. Then $T(H, \chi) T_{t^{+1}}=T_{t+1}, 1 \leqq j \leqq \chi(i d)$.

It follows from the orthogonality relations that the $T_{i}$ are annihilating idempotents, $1 \leqq i \leqq \lambda(i d)$. In particular, $T(G, \lambda) T_{i}=T_{i}$. Could $T(H, \chi) T(G, \lambda)=0$ ? If so, then $0=T(H, \chi) T(G, \lambda) T_{i}=T(H, \chi) T_{i}, 1 \leqq$ $i \leqq \lambda(i d)$. But then $T_{t^{+}}=0,1 \leqq j \leqq \chi(i d)$. We proceed to show that this is impossible.

Since $T_{t}$ is idempotent, its rank is equal to its trace;

$$
\text { trace } T_{i}=\frac{\lambda(i d)}{o(G)} \sum_{\sigma \in G} \ell_{i i}(\sigma) \rho(\sigma),
$$

where $\rho(\sigma)$ is the character of the representation $\sigma \rightarrow P(\sigma)$. Since $\rho$ is the restriction to $G$ of a character of $S_{m}$, it is real. Again employing the orthogonality relations, the trace of $T_{i}$ becomes $(\lambda, \rho)_{G}$. In particular, each of the $T_{i}$ has equal rank. A similar calculation shows that rank $T(G, \lambda)=\lambda(i d)(\lambda, \rho)_{G}$. But since $m \leqq n$, it is known ([13], [11], or [3]) that $\dot{T}(G, \lambda) \neq 0$. Therefore, $(\lambda, \rho)_{G} \neq 0$ and $T_{1} \neq 0,1 \leqq i \leqq \lambda(i d)$.

As we observed above,

$$
1_{\otimes}=\sum_{\zeta \in \mathscr{Y}(H)} T(H, \zeta)=\sum_{\xi \in \mathcal{A}(G)} T(G, \xi) .
$$


Therefore, if $\chi \in \mathscr{I}(H)$,

$$
\begin{aligned}
T(H, \chi) & =T(H, \chi) l_{\otimes} \\
& =T(H, \chi) \sum_{\xi \in \mathscr{G}(G)} T(G, \xi) \\
& =T(H, \chi) \theta_{1}=\theta_{1} T(H, \chi),
\end{aligned}
$$

where $\theta_{1}=\Sigma T(G, \xi)$; the sum being over those $\xi \in \mathscr{I}(G)$ such that $(\chi, \xi)_{H} \neq 0$.

Similarly, if $\lambda \in \mathscr{I}(G)$ and $\left.\lambda\right|_{H}=a_{1} \chi_{1}+\cdots+a_{r} \chi_{r}$, then

$$
\begin{aligned}
T(G, \lambda) & =T(G, \lambda) 1_{\otimes} \\
& =T(G, \lambda) \sum_{\zeta \in \mathscr{\mathscr { S }}(H)} T(H, \zeta) \\
& =T(G, \lambda) \theta_{2}=\theta_{2} T(G, \lambda),
\end{aligned}
$$

where

$$
\theta_{2}=\sum_{i=1}^{r} T\left(H, \chi_{i}\right)
$$

Lemma 3. (a) The operator $\theta_{1}-T(H, \chi)$ is an orthogonal projection. If $n \geqq m, \theta_{1}=T(H, \chi)$ if and only if $\left.\xi\right|_{H}$ is a multiple of $\chi$ for all those $\xi \in \mathscr{I}(G)$ which satisfy $(\chi, \xi)_{H} \neq 0$. (b) The operator $\theta_{2}$ $T(G, \lambda)$ is an orthogonal projection. If $n \geqq m, \theta_{2}=T(G, \lambda)$ if and only if $\left(\chi_{i}, \xi\right)_{H}=0,1 \leqq i \leqq r$, for every $\xi \in \mathscr{I}(G)$ different from $\lambda$.

Proof. Each of $\theta_{1}, \theta_{2}$ is a sum of pairwise annihilating orthogonal projections and is, therefore, an orthogonal projection. That $\theta_{1}$ $T(H, \chi)$ and $\theta_{2}-T(G, \lambda)$ are orthogonal projections now follows from (11) and (12), respectively [4, pp. 148-149].

If $T(H, \chi)=\theta_{1}$, then $0=T(H, \zeta) T(H, \chi)=T(H, \zeta) \theta_{1}$, for every $\zeta \in \mathscr{I}(H)$ different from $\chi$. But, by Lemma $1, T(H, \zeta) \theta_{1}=0$ if and only if $T(H, \zeta) T(G, \xi)=0$ for every $\xi \in \mathscr{I}(G)$ such that $(\chi, \xi)_{H} \neq 0$. By Lemma $2, T(H, \zeta) T(G, \xi)=0$ if and only if $\left.\zeta \notin \xi\right|_{H}$. Thus, none of the $\xi$ involved in $\theta_{1}$ contains, upon restriction to $H$, an irreducible character $\zeta$ different from $\chi$, i.e., for every $\xi$ involved in $\theta_{1},\left.\xi\right|_{H}=\xi($ id $) \chi / \chi(i d)$.

If $T(G, \lambda)=\theta_{2}$, let $\xi \in \mathscr{I}(G)$ be different from $\lambda$. Then $0=$ $T(G, \xi) T(G, \lambda)=T(G, \xi) \theta_{2}$. Again by Lemma 1, it follows that $T(G, \xi) T\left(H, \chi_{\imath}\right)=0,1 \leqq i \leqq r$. Appealing to Lemma 2 , we find that $\left.\chi_{1} \notin \xi\right|_{H}$.

We require one more result before we can prove Theorems 1 and 2 . 
Lemma 4. Suppose $A=\left(a_{i j}\right) \geqq 0$ is $m$-square. Take $n \geqq m$. Let $v_{1}, \cdots, v_{m} \in V$ be such that $a_{i j}=\left(v_{i}, v_{1}\right)$. Then

$$
\frac{\lambda(i d)}{o(G)} d_{\lambda}^{G}(A)=\left(T(G, \lambda) v_{1} \otimes \cdots \otimes v_{m}, v_{1} \otimes \cdots \otimes v_{m}\right)
$$

This is a standard result the proof of which is a straightforward computation relying only on the definitions.

Proof of Theorem 1. Assume $a_{i j}=\left(v_{l}, v_{i}\right)$. From Lemma 3, $\theta_{1}-T(H, \chi) \geqq 0$. Therefore,

$$
\left(\left[\theta_{1}-T(H, \chi)\right] v_{1} \otimes \cdots \otimes v_{m}, v_{1} \otimes \cdots \otimes v_{m}\right) \geqq 0,
$$

or

$$
(1 / o(G)) \Sigma \xi(i d) d_{\xi}^{G}(A) \geqq(\chi(i d) / o(H)) d_{\chi}^{H}(A)
$$

by Lemma 4 , where the summation is over those $\xi \in \mathscr{I}(G)$ such that $(\chi, \xi)_{H} \neq 0$. If equality holds, for all $A \geqq 0$, then equality holds in (13) for all $v_{1} \otimes \cdots \otimes v_{m}$. But these tensors span $\otimes^{m} V$. It follows (since $\left.\theta_{1}-T(H, \chi) \geqq 0\right)$ that $\theta_{1}=T(H, \chi)$. The case of equality in Theorem 1 then follows from the case of equality in Lemma $3 a$.

The proof of Theorem 2 is analogous.

Our work has also led us to some results involving symmetry classes of tensors.

Definition. Let $G$ be a subgroup of $S_{m}$. Let $\lambda \in \mathscr{I}(G)$. The range, $V_{\lambda}(G)$ of $T(G, \lambda)$ is called the symmetry class of tensors arising from $G$ and $\lambda$.

THEOREM 3. Let $H$ be a subgroup of $G$. Let $\chi \in \mathscr{I}(H)$. Then

$$
V_{\chi}(H) \subset \Sigma V_{\xi}(G)
$$

where the (direct) sum is over those $\xi \in \mathscr{I}(G)$ such that $\left.\chi \in \xi\right|_{H}$. If in addition $m \leqq n$, then equality holds if and only if $\left.\xi\right|_{H}$ is a multiple of $\chi$ for all $\xi \in \mathscr{I}(G)$ such that $\left.\chi \in \xi\right|_{H}$.

Proof. This is an immediate consequence of Lemma 3(a). (That the sum is direct follows from the Freese-Pierce-Wade result.) 
THEOREM 4. Let $H$ be a subgroup of $G$. Let $\lambda \in \mathscr{I}(G)$. Suppose $\left.\lambda\right|_{H}=a_{1} \chi_{1}+\cdots+a_{r} \chi_{r}$, where $a_{1}, \cdots, a_{r}$ are nonnegative integers and $\chi_{1}, \cdots, \chi_{r} \in \mathscr{I}(H)$. Then

$$
V_{\lambda}(G) \subset \sum_{i=1}^{r} V_{\chi_{i}}(H)
$$

where the sum is direct. If $m \leqq n$, then equality holds if and only if $\left(\chi_{i}, \xi\right)_{H}=0,1 \leqq i \leqq r$, for every $\xi \in \mathscr{I}(G)$ different from $\lambda$.

Proof. This follows from Lemma 3(b).

\section{REFERENCES}

1. Gernot M. Engel, Regular sets of matrices for generalized matrix functions, Linear Algebra Appl., 7 (1973). 243-274.

2. Walter Feit, Characters of Finite Groups, Benjamin, New York-Amsterdam, 1967.

3. Ralph Freese, Inequalities for generalized matrix functions based on arbitrary characters, Linear Algebra Appl., 7 (1973), 337-345.

4. P. R. Halmos, Finite Dimensional Vector Spaces, 2nd ed., Van Nostrand, Princeton, N.J., 1958.

5. Marvin Marcus, Finite Dimensional Multilinear Algebra, Part 1, Marcel Dekker, New York, 1973.

6. Russell Merris, A dominance theorem for partitioned hermitian matrices, Trans. Amer. Math. Soc., 164 (1972), 341-352.

7. - An identity for matrix functions, Pacific J. Math., 50 (1974), 557-562.

8. - An identity involving generalized matrix functions, Linear and Multilinear Algebra, 2 (1974), 123-125.

9. Russell Merris and William Watkins, Character induced subgroups, J. Research (U.S.) National Bureau of Standards 77 B (1973), 93-99.

10. Morris Newman, Matrix Representations of Groups, National Bureau of Standards Applied Math. Series 60, Superintendent of Documents, Washington D.C., 1968.

11. Stephen Pierce, Orthogonal decompositions of tensor spaces, J. Research (U.S.) National Bureau of Standards 74 B (1970), 41-44.

12. I. Schur, Über endliche Gruppen und Hermitesche Formen, Math. Z., 1 (1918), 184-207.

13. T. L. Wade, Tensor algebra and Young's symmetry operators, Amer. J. Math., 63 (1941), 645-657.

14. S. G. Williamson, On a class of combinatorial inequalities, J. Combinatorial Theory, 6 (1969), 359-369.

Received July 23, 1975. The research leading to this article was supported in part by the National Science Foundation under Grant No. MPS 75-05799.

California State University, Hayward 




\section{Pacific Journal of Mathematics}

\section{Vol. 62, No. $1 \quad$ January, 1976}

Mieczyslaw Altman, Contractor directions, directional contractors and

directional contractions for solving equations . .................. 1

Michael Peter Anderson, Subgroups of finite index in profinite groups .........

Zvi Arad, Abelian and nilpotent subgroups of maximal order of groups of odd order

John David Baildon and Ruth Silverman, On starshaped sets and Helly-type theorems ..........................................

John W. Baker and R. C. Lacher, Some mappings which do not admit an

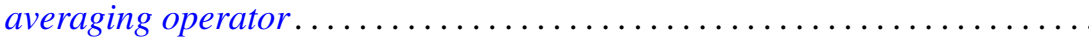

Joseph Barback, Composite numbers and prime regressive isols . . . . . . . . . .

David M. Boyd, Composition operators on $H^{p}(A) \ldots \ldots \ldots \ldots \ldots \ldots \ldots$

Maurice Chacron, Co-radical extension of PI rings . . . . . . . . . . . . .

Fred D. Crary, Some new engulfing theorems . . . . . . . . . . . . . . .

Victor Dannon and Dany Leviatan, A representation theorem for convolution transform with determining function in $L^{p} \ldots \ldots \ldots \ldots \ldots \ldots \ldots \ldots \ldots \ldots \ldots \ldots \ldots \ldots$

Mahlon M. Day, Lumpy subsets in left-amenable locally compact

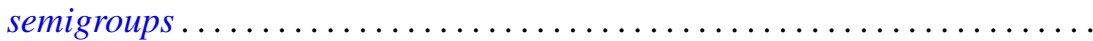

Michael A. Gauger, Some remarks on the center of the universal enveloping algebra of a classical simple Lie algebra . .

David K. Haley, Equational compactness and compact topologies in rings

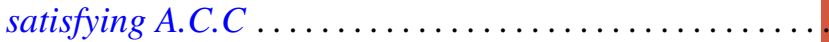

Raymond Heitmann, Generating ideals in Prüfer domains .

Gerald Norman Hile, Entire solutions of linear elliptic equations with

Laplacian principal part. .

Richard Oscar Hill, Moore-Postnikov towers for fibrations in which $\pi_{1}$ (fiber) is non-abelian

John Rast Hubbard, Approximation of compact homogeneous maps . .

Russell L. Merris, Relations among generalized matrix functions . .

V. S. Ramamurthi and Edgar Andrews Rutter, On cotorsion radicals ...

Ralph Tyrrell Rockafellar and Roger Jean-Baptiste Robert Wets, Stochastic

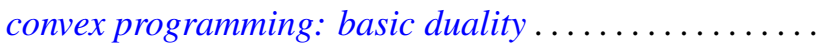

Alban J. Roques, Local evolution systems in general Banach spaces ..

I. Bert Russak, An indirect sufficiency proof for problems with bounded state variables.

Richard Alexander Sanerib, Jr., Ultrafilters and the basis property. .

H. A. Seid, The decomposition of multiplication operators on $L_{p}$-spaces . .

Franklin D. Tall, The density topology .................. 